\title{
Analysis of a mathematical model of the dynamics of contagious bovine pleuropneumonia
}

\author{
Achamyelesh Amare Aligaz, Justin Manango W. Munganga
}

\begin{abstract}
Contagious bovine pleuropneumonia (CBPP) is a disease of cattle and water buffalo caused by Mycoplasma mycoides subspecies mycoides (Mmm). It attacks the lungs and the membranes that line the thoracic cavity. The disease is transmitted by inhaling droplets disseminated through coughing by infected cattle. In this paper a deterministic mathematical model for the transmission of Contagious Bovine plueropnemonia is presented. The model is a five compartmental model consisting of susceptible, Exposed, Infectious, Persistently infected and Recovered compartments. We derived a formula for the basic reproduction number $\mathscr{R}_{0}$. For $\mathscr{R}_{0} \leq 1$, the disease free equilibrium is globally asymptotically stable, thus CBPP dies out; whereas for $\mathscr{R}_{0}>1$, the unique endemic equilibrium is globally asymptotically stable and hence the disease persists. Elasticity indices for $\mathscr{R}_{0}$ with respect to different parameters are calculated; indicating parameters that are important for control strategies to bring $\mathscr{R}_{0}$ below 1 , the effective contact rate $\beta$ has the largest elasticity index. As the disease control options are associated to these parameters, for some values of these parameters, $\mathscr{R}_{0}<1$, thus the disease can be controlled.
\end{abstract}

Achamyelesh Amare Aligaz

Department of Mathematical Sciences, University of South Africa

Justin Manango W. Munganga

Department of Mathematical Sciences, University of South Africa

e-mail: munga jmw@unisa.ac.za

Citation: Achamyelesh Amare Aligaz, Justin Manango W. Munganga, Analysis of a mathematical model of the dynamics of contagious bovine pleuropneumonia, in R. Anguelov, M. Lachowicz (Editors), Mathematical Methods and Models in Biosciences, Biomath Forum, Sofia, 2018, pp. 64-80,http://dx.doi.org/10.11145/texts.2017.12.253

Copyright: (c) 2018 Aligaz et al. This article is distributed under the terms of the Creative Commons Attribution License (CC BY 4.0) which permits unrestricted use, distribution, and reproduction in any medium, provided the original author and source are credited. 


\section{Introduction}

Contagious bovine pleuropneumonia (CBPP) is a disease of cattle and water buffalo caused by Mycoplasma mycoides subspecies mycoides (Mmm). It attacks the lungs and the membranes that line the thoracic cavity. CBPP is manifested by loss of appetite, fever and respiratory signs, such as laboured or rapid respiratory rate, cough and nasal discharges. In hot climates, an affected animal often stands by itself in the shade, its head lowered and extended, its back slightly arched, and its limbs turned out. CBPP is characterized by its variable course and insidious nature [6]. In many cases, the disease progresses rapidly causing animals lose, and breathing becomes very laboured, with a grunt at expiration. The animals become recumbent (lie down) and in severe cases die after 1-3 weeks. It impacts cattle production in sub-Saharan Africa [9]. CBPP is currently considered one of the main stumbling blocks to the growth of the livestock industry on the African continent [17].

Transmission of the disease occurs through direct contact between an infected and a susceptible animal which becomes infected by inhaling droplets disseminated by coughing. Since some animals can carry the disease without showing signs of illness, controlling the spread is more difficult.

Historically, CBPP was a disease of Europe and Asia [18]. It was known in Europe in 1773 and it spread almost to all parts of the world in the nineteenth century through the cattle trade [2]. CBPP was introduced to Africa in the colonial era and subsequently spread throughout the continent [3]. Through the application of restrictions of the movement of cattle, as well as test and slaughter policies combined with compensation for livestock keepers, CBPP has been eradicated from Australia, Europe, Asia, and America. However, such policies are difficult to apply in most African countries because of pastoralism, lack of economic resources, and fragmented veterinary services [11]. As a result, the disease remains endemic in Africa particularly in tropical and subtropical regions (West, Central, East, and parts of Southern Africa) of the continent. Its incidence began to decline in Africa by the 1970s; however, this disease increased in prevalence in endemic areas and re-emerged in some African and European countries that had been CBPP free [1].

It is a prominent cattle disease in Africa, where outbreaks of the disease reported from 20 countries in 2006, with the highest number of cases in Ethiopia, Angola, and Cameroon [8]. Over the last few years, major CBPP epidemics have been experienced in Eastern, Southern, and West Africa. Currently, it affects 27 countries in Africa with an estimated annual cost of 2 billion dollar [10]. A total of 2,719 outbreaks were reported in Africa between 1995 and 2002 [1]. The control of CBPP in sub-Saharan Africa, from the Zambian perspective, includes failure in the delivery of veterinary services, lack of a cattle identification system, natural phenomenon, livestock husbandry systems in the traditional sector, human movements, traditional practices among cattle farmers and cattle marketing systems [7].

The Pan African programme for the Control of epizootics (PACE) identified CBPP as the second most important transboundary disease in Africa, after Rinderpest [15]. Following the eradication of rinderpest from Africa, CBPP has become the disease of prime concern in terms of epizootics that affect cattle in the con- 
tinent. It is estimated that annual loss due to CBPP amount to 38.81 million US dollars in 12 endemically infected Sub-Saharan African countries [15]. On account of its transmissibility and economic impacts, CBPP is now recognized as a priority transboundary disease and has thus been categorized as the only bacterial disease in the world organization for animal health (OIE) list A diseases [1].

Mathematical models provide the means to generate evidence based information on infectious disease control and play an important role in understanding the dynamics of infectious diseases. As in [14], modelling techniques are used to assess the potential impact of early elimination of infected cattle via accurate diagnosis on CBPP dynamics. Generally, the model predicts that regular testing and elimination of positive reactors using improved tests will play a significant role in minimizing CBPP burden especially in the current situation where improved vaccines are yet to be developed.

A mathematical model of CBPP is also used to compare economic efficiency of local (i.e., at the herd level) CBPP-control strategies (vaccination and antibiotic treatments). A study conducted in Ethiopian highlands cattle-smallholder system (Boji district, West Wellega Zone), with low CBPP virulence, revealed that antibiotics were the most economically efficient strategy than vaccination [4].

In this paper, a deterministic mathematical model for the transmission of CBPP dynamics is presented. We derived the formula for the basic reproduction number $\mathscr{R}_{0}$, and determined the stability of the disease free and the endemic equilibrium in terms of $\mathscr{R}_{0}$. Sensitivity analysis of $\mathscr{R}_{0}$ with respect to the parameter that it depends on and it is found that $\mathscr{R}_{0}$ is very sensitive to the effective contact rate $\beta$, which also have large regression coefficient in [18], the rate of recovery of persistently infected cattle $\psi$, which has the least regression coefficient in [6], and the rate of recovery of infectious cattle $\alpha_{r}$; but less sensitive to the death/birth rate $\mu$; in contrary, it has large regression coefficient in [6], the rate of infection reactivation of persistently infected cattle $k$, and the rate of transition from exposed to infectious class $\gamma$. Unlikely to [6], it is independent of the initial value of the system. Numerical simulation is performed using MATLAB.

The rest of the paper is structured as follows. In section 2, we present a mathematical modelling of the dynamics of CBPP. In section 3, we prove the well-posedness of the system. In section 4 , we calculate equilibria of the system and derive a formula of the basic reproduction number $\mathscr{R}_{0}$. In section 5, stability analysis of the DFE and EE is presented. In section 6, we present estimation of parameter values, elasticity index of parameters and numerical simulations. Lastly, in section 7, we present our conclusion and remarks.

\section{Mathematical model}

We model the transmission dynamics of Contagious Bovine Plueropnemonia (CBPP). The compartmental model with 5 classes is shown in Figure 1 . In this model we assume an open population where all new born animals are considered to be suscep- 
tible and added to the susceptible $(S)$ compartment with a constant recruitment rate $\Lambda$. Death due to contagious bovine plueropneumonia does not occur. Natural mortality occur at the rate $\mu$ and results in losses from all five compartments. The natural mortality rate and birth rate are assumed to be equal. Susceptible animals move to the exposed compartment $(E)$ at a rate of $\beta \frac{I}{N}$. Animals in the exposed compartment are infected but not infectious. The period in which animals stay infected without showing the symptom of the disease or without being infectious is said to be a latent period. Then, after the latent period, the animals in the exposed compartment move to the infectious compartment $(I)$ at a rate of $\gamma$. The infectious animals either heal and enter directly into the recovered $(R)$ compartment at a rate of $\alpha_{r}$ or they pass through a process of sequestration and enter into persistently infected $(Q)$ compartment at a rate of $\alpha_{q}$. Animals in persistently infected compartment are encapsulated and infected, but not infectious. As sequestra resolve and/or become non-infected, then the animals in persistently infected compartment move to the recovered $(R)$ compartment at a rate of $\psi$. Note that Animals in the recovered compartment can have sterile sequestra (a sequestra which is not infected). Whereas, Infected sequestra can occasionally be reactive and in this instance the animal will transition from the persistently infected $(Q)$ compartment back to the infectious $(I)$ compartment at a rate of $k$. And we assume random mixing of all individuals in the population. The total population size at time $t, N(t)$, is the sum of the numbers in the five compartments; i.e, $N(t)=S(t)+E(t)+I(t)+Q(t)+R(t)$; where

- $S(t)$ : number of susceptible animals at time $t$.

- $E(t)$ : number of exposed animals at time $t$.

- $I(t)$ : number of infectious animals at time $t$.

- $Q(t)$ : number of persistently infected animals at time $t$.

- $R(t)$ : number of recovered animals at time $t$.

And, we have the following parameters:

- $\alpha_{q}$ : The rate at which infectious animals move to persistently infected compartment

- $\alpha_{r}$ : Rate of recovery of infectious animals

- $\beta$ : Effective contact rate

- $\gamma$ : Transition rate from exposed to infectious compartment

- $k$ : The rate at which persistently infected animals move back to infectious compartment

- $\mu$ : Natural mortality rate

- $\omega$ : Rate of loss of natural immunity

- $\psi$ : Rate of recovery of persistently infected animals.

- $\Lambda$ : The constant recruitment rate.

Flow diagram of the model is as shown in Figure 1 


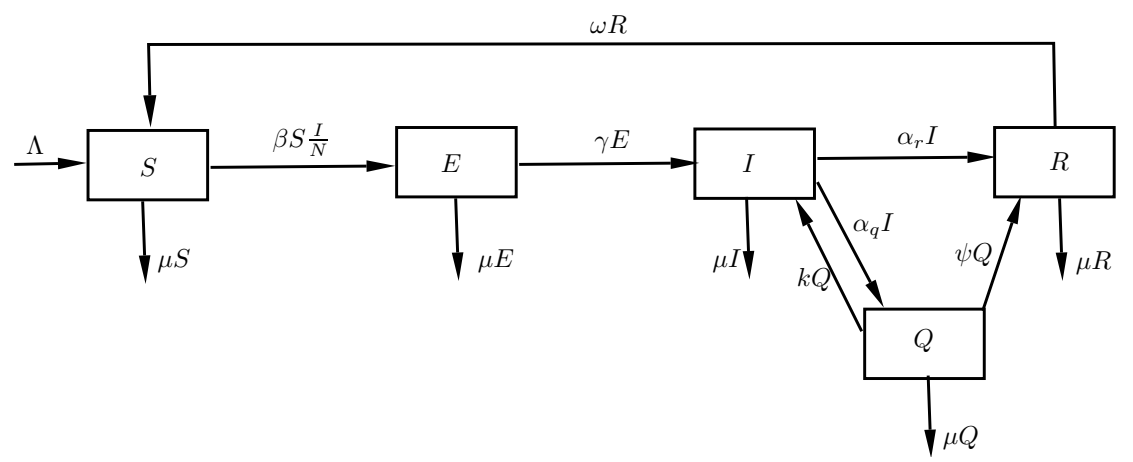

Fig. 1 A compartmental model for the dynamics of CBPP without any intervention

The compartmental model in Figure 1 can be written as a system of differential equations given by the system (1)- 5 )

$$
\begin{aligned}
& \frac{d S}{d t}=\Lambda-\beta S \frac{I}{N}+\omega R-\mu S \\
& \frac{d E}{d t}=\beta S \frac{I}{N}-\gamma E-\mu E \\
& \frac{d I}{d t}=\gamma E+k Q-\alpha_{r} I-\alpha_{q} I-\mu I \\
& \frac{d Q}{d t}=\alpha_{q} I-k Q-\psi Q-\mu Q \\
& \frac{d R}{d t}=\alpha_{r} I+\psi Q-\omega R-\mu R .
\end{aligned}
$$

Since $N$ is constant, equations (1) - (5) can be reduced to:

$$
\begin{aligned}
\frac{d S}{d t} & =\Lambda-\beta S \frac{I}{N}+\omega R-\mu S \\
\frac{d E}{d t} & =\beta S \frac{I}{N}-\gamma E-\mu E \\
\frac{d I}{d t} & =\gamma E+k Q-\alpha_{r} I-\alpha_{q} I-\mu I \\
\frac{d Q}{d t} & =\alpha_{q} I-k Q-\psi Q-\mu Q \\
R(t) & =N(t)-(S(t)+E(t)+I(t)+Q(t))
\end{aligned}
$$

Nonnegative initial conditions with $S(0)+E(0)+I(0)+Q(0)+R(0)$ positive and small complete the formulation of the model. 


\section{Well-posedness of the system}

Let $X(t)=(S(t), E(t), I(t), Q(t))$ and

$$
\begin{aligned}
f: \Gamma \longrightarrow \mathscr{Y} \\
X \longmapsto X^{\prime}
\end{aligned}
$$

such that $f=\left(f_{1}, f_{2}, f_{3}, f_{4}\right)$, where

$$
\begin{aligned}
& f_{1}(X)=\frac{d S}{d t}=\Lambda-\beta S \frac{I}{N}+\omega R-\mu S \\
& f_{2}(X)=\frac{d E}{d t}=\beta S \frac{I}{N}-\gamma E-\mu E \\
& f_{3}(X)=\frac{d I}{d t}=\gamma E+k Q-\alpha_{r} I-\alpha_{q} I-\mu I \\
& f_{4}(X)=\frac{d Q}{d t}=\alpha_{q} I-k Q-\psi Q-\mu Q .
\end{aligned}
$$

Then, equations (6) - 9) can be written of the form:

$$
X^{\prime}(t)=f(X(t)) ; \quad X(0)=\left(S_{0}, E_{0}, I_{0}, Q_{0}, R_{0}\right) \in \Gamma .
$$

Theorem 1. If $f$ is given by equation (15) and the initial conditions $X(0)=$ $\left(S_{0}, E_{0}, I_{0}, Q_{0}, R_{0}\right)$ is nonnegative, then the system (1)-(5) has a unique solution $X(t)$ which is nonnegative and bounded.

Proof. It is clear that the right hand of the system (1)-5 is $C^{1}$, thus, $f$ is differentiable and hence locally Lipschitz continuous in some open ball containing $X(0)$. Hence a unique solution $X(t)$ of the system (1)- (5) exists locally. For $X(0) \geq 0$, let $X(t)$ be the solution of (1)- 5 , and let $t_{0}$ be the smallest positive $t$ such that $S\left(t_{0}\right)=0$ or $I\left(t_{0}\right)=0$ or $E\left(t_{0}\right)=0$ or $Q\left(t_{0}\right)=0$ or $R\left(t_{0}\right)=0$. By continuity of $S, E, I, Q$ and $R$, there exists $t^{*}>t_{0}$ such that if $S\left(t_{0}\right)=0$, then clearly from (1) $S^{\prime}(t)=\Lambda+\omega R(t) \geq 0$, for $t \in\left[t_{0}, t^{*}\right]$. Similarly, if $E\left(t_{0}\right)=0$, then from 22, $E^{\prime}(t)=$ $\beta S \frac{I}{N} \geq 0$, for $t \in\left[t_{0}, t^{*}\right]$. If $I\left(t_{0}\right)=0$, then from $33, I^{\prime}(t)=\gamma E(t)+k Q(t) \geq 0$, for $t \in\left[t_{0}, t^{*}\right]$. If $Q\left(t_{0}\right)=0$, then from (4), $Q^{\prime}(t)=\alpha_{q} I(t) \geq 0$, for $t \in\left[t_{0}, t^{*}\right]$, and if $R\left(t_{0}\right)=0$, then from (5), $R^{\prime}(t)=\alpha_{r} I(t)+\psi Q(t) \geq 0$, for $t \in\left[t_{0}, t^{*}\right]$. By Proposition B.7. of [13], the solution of the system (1)-(5] is nonnegative. Finally, $S(t) \leq N, E(t) \leq N, I(t) \leq N, Q(t) \leq N$ and $Q(t) \leq N$ for all $t \geq 0$. Thus $X$ is bounded. 


\section{Equilibria and basic reproduction number}

\subsection{Equilibria of the system}

Equilibria are points where the variables do not change with time: i.e.,

$$
\frac{d S}{d t}=\frac{d E}{d t}=\frac{d I}{d t}=\frac{d Q}{d t}=\frac{d R}{d t}=0
$$

Let $\left(S^{*}, E^{*}, I^{*}, Q^{*}, R^{*}\right)$ be the equilibria of the system (1)- 5 with the left hand side equal zero,

$$
\begin{aligned}
\frac{d S}{d t} & =\Lambda-\beta S^{*} \frac{I^{*}}{N}+\omega R^{*}-\mu S^{*}=0 \\
\frac{d E}{d t} & =\beta S^{*} \frac{I^{*}}{N}-\gamma E^{*}-\mu E^{*}=0 \\
\frac{d I}{d t} & =\gamma E^{*}+k Q^{*}-\alpha_{r} I^{*}-\alpha_{q} I^{*}-\mu I^{*}=0 \\
\frac{d Q}{d t} & =\alpha_{q} I^{*}-k Q^{*}-\psi Q^{*}-\mu Q^{*}=0
\end{aligned}
$$

From equation (19),

$$
Q^{*}=\frac{\alpha_{q} I^{*}}{\bar{\mu}_{k \psi}} .
$$

Putting equation (20) into equation (18), we get

$$
E^{*}=\frac{\left(\bar{\mu}_{\alpha} \bar{\mu}_{k \psi}-k \alpha_{q}\right) I^{*}}{\gamma \bar{\mu}_{k \psi}},
$$

where $\bar{\mu}_{\alpha}=\alpha_{r}+\alpha_{q}+\mu, \bar{\mu}_{k \psi}=k+\psi+\mu$

Since $\Lambda=\mu N$, putting 21] into equation (17) yields $I^{*}=0$, or

$$
S^{*}=\frac{(\gamma+\mu)\left(\bar{\mu}_{\alpha} \bar{\mu}_{k \psi}-k \alpha_{q}\right) N}{\gamma \beta \bar{\mu}_{k \psi}} .
$$

When $I^{*}=0$, we get $Q^{*}=E^{*}=0$ (from equation 20 and 21). And, from equation 16 .

$$
\begin{aligned}
& -\beta S^{*} \frac{I^{*}}{N}+\Lambda+\omega R^{*}-\mu S^{*}=0 \\
& \Leftrightarrow \Lambda+\omega\left(N-\left(S^{*}+E^{*}+I^{*}+Q^{*}\right)\right)-\mu S^{*}=0 \quad\left(\text { Since } I^{*}=0\right) \\
& \Leftrightarrow \Lambda+\omega\left(N-S^{*}\right)-\mu S^{*}=0 \quad\left(\text { since } E^{*}=I^{*}=Q^{*}=0\right) \\
& \Leftrightarrow \Lambda+\omega N-\omega S^{*}-\mu S^{*}=0 \\
& \Leftrightarrow S^{*}=N .
\end{aligned}
$$


Hence, $(N, 0,0,0)$ is the disease free equilibrium.

For the other case, when $S^{*}=\frac{(\gamma+\mu)\left(\bar{\mu}_{\alpha} \bar{\mu}_{k \psi}-k \alpha_{q}\right) N}{\gamma \beta \bar{\mu}_{k \psi}}$, we use equations $16, \sqrt{20}, 21$, and (22) together with the equality $R^{*}=N-\left(S^{*}+E^{*}+I^{*}+Q^{*}\right)$, yields

$$
I^{*}=\frac{\gamma \beta(\omega+\mu) \bar{\mu}_{k \psi}-(\omega+\mu)(\gamma+\mu)\left(\bar{\mu}_{k \psi} \bar{\mu}_{\alpha}-k \alpha_{q}\right)}{\beta(\gamma+\mu+\omega)\left(\bar{\mu}_{\alpha} \bar{\mu}_{k \psi}-k \alpha_{q}\right)+\omega \gamma \beta\left(\bar{\mu}_{k \psi}+\alpha_{q}\right)} N .
$$

Hence,

$$
\left(S^{*}, E^{*}, I^{*}, Q^{*}\right)=\left(\frac{(\gamma+\mu)\left(\bar{\mu}_{\alpha} \bar{\mu}_{k \psi}-k \alpha_{q}\right) N}{\gamma \beta \bar{\mu}_{k \psi}}, \frac{\left(\bar{\mu}_{\alpha} \bar{\mu}_{k \psi}-k \alpha_{q}\right) I^{*}}{\gamma \bar{\mu}_{k \psi}}, I^{*}, \frac{\alpha_{q} I^{*}}{\bar{\mu}_{k \psi}}\right)
$$

is the endemic equilibrium.

\subsection{Basic reproduction number $\left(\mathscr{R}_{0}\right)$}

The basic reproduction number refers to the total number of animals infected by a single infectious animal through out its infectious period. We say that the disease die out if $\mathscr{R}_{0} \leq 1$ and persists in the population if $\mathscr{R}_{0}>1$.

We use the next generation matrix as presented in [16], to calculate the basic reproduction number. Compartments $E, I$ and $Q$ are considered to be the disease compartments and $S$ is the non-disease compartment. We set $\mathscr{F}=\left(\mathscr{F}_{1}, \ldots, \mathscr{F}_{4}\right)^{T}$ and $\mathscr{V}=\left(\mathscr{V}_{1}, \ldots, \mathscr{V}_{4}\right)^{T}$, where $\mathscr{F}_{i}$ represents the rate of new infections in the $i^{\text {th }}$ disease compartment, $\mathscr{V}_{i}^{+}(X)$ being the transfer rate of individuals into compartment $i$ by all other means while $\mathscr{V}_{i}^{-}(X)$ represents the transfer rate of individual out of compartment $i$. Assuming $X_{0}$ to be the DFE, we have

$$
\begin{gathered}
\mathscr{F}=\left(\begin{array}{c}
\frac{\beta S I}{N} \\
0 \\
0
\end{array}\right), \quad \mathscr{V}=\mathscr{V}^{-}-\mathscr{V}^{+}=\left(\begin{array}{c}
(\gamma+\mu) E \\
\bar{\mu}_{\alpha} I-\gamma E-k Q \\
\bar{\mu}_{k \psi} Q-\alpha_{q} I
\end{array}\right), \\
F=\left(\frac{\partial \mathscr{F}_{i}}{\partial x_{j}}\left(X_{0}\right)\right)=\left(\begin{array}{lll}
0 & \beta & 0 \\
0 & 0 & 0 \\
0 & 0 & 0
\end{array}\right), \quad V=\left(\frac{\partial \mathscr{V}_{i}}{\partial x_{j}}\left(X_{0}\right)\right)=\left(\begin{array}{ccc}
\gamma+\mu & 0 & 0 \\
-\gamma & \bar{\mu}_{\alpha} & -k \\
0 & -\alpha_{q} & \bar{\mu}_{k \psi}
\end{array}\right)
\end{gathered}
$$

and 


$$
V^{-1}=\left(\begin{array}{ccc}
\frac{1}{\gamma+\mu} & 0 & 0 \\
\frac{\gamma \bar{\mu}_{k \psi}}{(\gamma+\mu)\left(\bar{\mu}_{k \psi} \bar{\mu}_{\alpha}-\alpha_{q} k\right)} & \frac{\bar{\mu}_{k \psi}}{\left(\bar{\mu}_{k \psi} \bar{\mu}_{\alpha}-\alpha_{q} k\right)} \frac{k}{\left(\bar{\mu}_{k \psi} \bar{\mu}_{\alpha}-\alpha_{q} k\right)} \\
\frac{\alpha_{q} \gamma}{(\gamma+\mu)\left(\bar{\mu}_{k \psi} \bar{\mu}_{\alpha}-\alpha_{q} k\right)} \frac{\alpha_{q}}{\left(\bar{\mu}_{k \psi} \bar{\mu}_{\alpha}-\alpha_{q} k\right)} \frac{\bar{\mu}_{\alpha}}{\left(\bar{\mu}_{k \psi} \bar{\mu}_{\alpha}-\alpha_{q} k\right)}
\end{array}\right)
$$

So,

$$
F V^{-1}=\left(\begin{array}{ccc}
\frac{\beta \gamma \bar{\mu}_{k \psi}}{(\gamma+\mu)\left(\bar{\mu}_{k \psi} \bar{\mu}_{\alpha}-\alpha_{q} k\right)} & 0 & 0 \\
0 & 0 & 0 \\
0 & 0 & 0
\end{array}\right)
$$

and

$$
\mathscr{R}_{0}=\rho\left(F V^{-1}\right)=\frac{T}{2}+\sqrt{\left(\frac{T}{2}\right)^{2}-D},
$$

where, $T$ and $D$ are trace and determinant of the matrix $F V^{-1}$. Since $D=0$,

$$
\mathscr{R}_{0}=T=\frac{\beta \gamma \bar{\mu}_{k \psi}}{(\gamma+\mu)\left(\bar{\mu}_{k \psi} \bar{\mu}_{\alpha}-\alpha_{q} k\right)},
$$

where $\frac{\gamma}{(\gamma+\mu)}$ is the probability that a newly infected individual survives the latent period and $\frac{1}{\bar{\mu}_{\alpha}}$ is the average adjusted infectious period. Thus, $\mathscr{R}_{0}$ is the number of secondary infections caused by an infectious cattle during its infectious period.

\section{Stability analysis}

\subsection{Stability analysis of the Disease Free Equilibrium (DFE)}

\subsubsection{Local stability analysis of the DFE}

Theorem 2. If $X_{0}$ is a DFE of the model given by equation (15), then $X_{0}$ is locally asymptotically stable if $\mathscr{R}_{0}<1$, and unstable if $\mathscr{R}_{0}>1$, where $\mathscr{R}_{0}$ is defined by equation (23).

Proof. see [16]. 


\subsubsection{Global stability analysis of the DFE}

Theorem 3. The disease free equilibrium, $(N, 0,0,0))$, is globally asymptotically stable in $\Omega$ if $\mathscr{R}_{0} \leq 1$ and unstable if $\mathscr{R}_{0}>1$, where

$$
\Omega=\left\{(S, E, I, Q) \in R_{+}^{4}: S \geq 0, E \geq 0, I \geq 0, Q \geq 0, S+E+I+Q \leq N\right\} .
$$

Proof. We use matrix-theoretic method as explained in [12]. We assume $x=$ $(E, I, Q)^{T}$ and $y=S$. And, considering $F, V$ and $V^{-1}$ as in Section 4.2 we set $f(x, y)=(F-V) x-\mathscr{F}(x, y)+\mathscr{V}(x, y)=\left(\begin{array}{c}\beta I\left(1-\frac{S}{N}\right) \\ 0 \\ 0\end{array}\right)$.

And $V^{-1} F=\left(\begin{array}{lll}0 & \frac{\beta}{\gamma+\mu} & 0 \\ 0 & \frac{\beta \gamma \bar{\mu}_{k \psi}}{(\gamma+\mu)\left(\bar{\mu}_{k \psi} \bar{\mu}_{\alpha}-\alpha_{q} k\right)} & 0 \\ 0 & \frac{\beta \alpha_{q} \gamma}{(\gamma+\mu)\left(\bar{\mu}_{k \psi} \bar{\mu}_{\alpha}-\alpha_{q} k\right)} & 0\end{array}\right)$.

We observe that $F \geq 0, V^{-1} \geq 0, f(x, y) \geq 0, f(x, N)=0$ in $\Omega$; but the matrix $V^{-1} F$ is reducible. Thus we use Theorem 2.1 of [12] to construct a Lyapunov function. Denote the left eigenvector of $V^{-1} F$ corresponding to the eigenvalue $\mathscr{R}_{0}$ by $\left(v_{1}, v_{2}, v_{3}\right)$. Then

$$
\left(v_{1}, v_{2}, v_{3}\right) V^{-1} F=\mathscr{R}_{0}\left(v_{1}, v_{2}, v_{3}\right) \text {, }
$$

or

$$
\left(v_{1}, v_{2}, v_{3}\right) V^{-1} F=\left(0, \frac{\beta\left(\bar{\mu}_{k \psi} \bar{\mu}_{\alpha}-\alpha_{q} k\right) v_{1}+\beta \gamma \bar{\mu}_{k \psi} v_{2}+\beta \alpha_{q} \gamma v_{3}}{(\gamma+\mu)\left(\bar{\mu}_{k \psi} \bar{\mu}_{\alpha}-\alpha_{q} k\right)}, 0\right)
$$

and

$$
\mathscr{R}_{0}\left(v_{1}, v_{2}, v_{3}\right)=\frac{\beta \gamma \bar{\mu}_{k \psi}}{(\gamma+\mu)\left(\bar{\mu}_{k \psi} \bar{\mu}_{\alpha}-\alpha_{q} k\right)}\left(v_{1}, v_{2}, v_{3}\right)
$$

Thus, from equation 24 - 25), one solution is given by $v_{1}=0, v_{3}=0$ and $v_{2}=$ $\frac{1}{(\gamma+\mu)\left(\bar{\mu}_{k \psi} \bar{\mu}_{\alpha}-\alpha_{q} k\right)}$, thus $\omega^{T}=\left(0, v_{2}, 0\right)$. Hence, by Theorem 2.1 of [12], we have

$$
Q=\omega^{T} V^{-1} x=\gamma \bar{\mu}_{k \psi} E+\bar{\mu}_{k \psi}(\gamma+\mu) I+k(\gamma+\mu) Q
$$

is the Lyapunov function for the system when $\mathscr{R}_{0} \leq 1$.

Since $Q^{\prime}=\left(\mathscr{R}_{0}-1\right) \omega^{T} x-\omega^{T} V^{-1} f(x, y)=0$ implies that $\mathscr{R}_{0}=1, x=0$ and $y=N$, $(N, 0,0,0)$ is the only invariant set in $\Omega$ when $x=0$ and $y=N$. Thus, by LaSalle's invariance principle, the $\operatorname{DFE}(N, 0,0,0)$ is globally asymptotically stable in $\Omega$ when $\mathscr{R}_{0} \leq 1$. 


\subsection{Global stability analysis of the Endemic Equilibrium (EE)}

Theorem 4. With $\Omega$ given as in Theorem 3 if $\mathscr{R}_{0}>1$ and $\omega=0$, then the unique endemic equilibrium $E^{*}$ of the equations (15) is globally asymptotically stable in the interior of $\Omega$.

Proof. We use a graph-theoretic method as in [12].

The weighted graph of the model given by system of equations $(1)-(5)$ is presented in figure 2

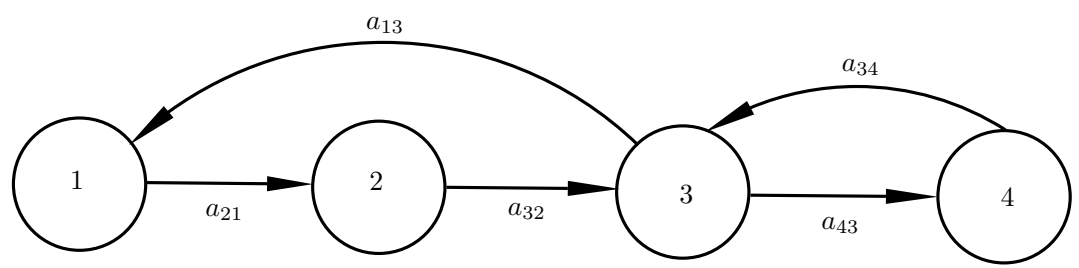

Fig. 2 The weighted digraph $(G, A)$ constructed for the model $1-5$

Set $D_{1}=S-S^{\star}-S^{\star} \ln \frac{S}{S^{\star}}, D_{2}=E-E^{\star}-E^{\star} \ln \frac{E}{E^{\star}}, D_{3}=I-I^{\star}-I^{\star} \ln \frac{I}{I^{\star}}, D_{4}=$ $Q-Q^{\star}-Q^{\star} \ln \frac{Q}{Q^{\star}}$ and $D_{5}=R-R^{\star}-R^{\star} \ln \frac{R}{R^{\star}}$.

From endemic equilibria calculation, we have $\Lambda=\frac{\beta S^{*} I^{*}}{N}-\omega R^{*}+\mu S^{*}, \gamma+\mu=$ $\frac{\beta S^{*} I^{*}}{N E^{*}}, \bar{\mu}_{\alpha}=\frac{\gamma E^{*}+k Q^{*}}{I^{*}}, \bar{\mu}_{k \psi}=\frac{\alpha_{q} I^{*}}{Q^{*}}$ and $\omega+\mu=\frac{\alpha_{r} I^{*}+\psi Q^{*}}{R^{*}}$. And also using the inequality $1-x+\ln x \leq 0$, differentiation with respect to $t$ gives 


$$
\begin{aligned}
& D_{1}^{\prime}=\left(1-\frac{S^{*}}{S}\right)\left(-\frac{\beta S I}{N}+\frac{\beta S^{*} I^{*}}{N}-\omega R^{*}+\mu S^{*}+\omega R-\mu S\right) \\
& \leq \frac{\beta S^{*} I^{*}}{N}\left(1-\frac{S I}{S^{*} I^{*}}-\frac{S^{*}}{S}+\frac{I}{I^{*}}\right) \\
& +\omega R^{*}\left(\frac{R}{R^{*}}-2-\ln \left(\frac{S^{*}}{S}\right)-\ln \left(\frac{R}{R^{*}}\right)+\frac{S^{*}}{S}\right)=: a_{13} G_{13}+a_{15} G_{15} \\
& D_{2}^{\prime}=\left(1-\frac{E^{*}}{E}\right)\left(\frac{\beta S I}{N}-\frac{\beta S^{*} I^{*} E}{N E^{*}}\right) \\
& \leq \frac{\beta S^{*} I^{*}}{N}\left(\frac{S I}{S^{*} I^{*}}-\frac{E}{E^{*}}+\ln \left(\frac{E}{E^{*}}\right)+\ln \left(\frac{S^{*}}{S}\right)-\ln \left(\frac{I}{I^{*}}\right)\right) \\
& =\frac{\beta S^{*} I^{*}}{N}\left(\frac{S I}{S^{*} I^{*}}-\frac{I}{I^{*}}+\ln \left(\frac{S^{*}}{S}\right)\right) \\
& +\frac{\beta S^{*} I^{*}}{N}\left(-\frac{E}{E^{*}}+\frac{I}{I^{*}}-\ln \left(\frac{I}{I^{*}}\right)+\ln \left(\frac{E}{E^{*}}\right)\right) \\
& =: a_{21} G_{21}+a_{23} G_{23} \\
& D_{3}^{\prime}=\left(1-\frac{I^{*}}{I}\right)\left(\gamma E+k Q-\frac{\gamma E^{*} I}{I^{*}}-\frac{k Q^{*} I}{I^{*}}\right) \\
& \leq \gamma E^{*}\left(\frac{E}{E^{*}}-\frac{I}{I^{*}}+\ln \left(\frac{I}{I^{*}}\right)-\ln \left(\frac{E}{E^{*}}\right)\right) \\
& +k Q^{*}\left(\frac{Q}{Q^{*}}-\frac{I}{I^{*}}+\ln \left(\frac{I}{I^{*}}\right)-\ln \left(\frac{Q}{Q^{*}}\right)\right)=: a_{32} G_{32}+a_{34} G_{34} \\
& D_{4}^{\prime}=\left(1-\frac{Q^{*}}{Q}\right)\left(\alpha_{q} I-\frac{\alpha_{q} I^{*} Q}{Q^{*}}\right) \\
& \leq \alpha_{q} I^{*}\left(\frac{I}{I^{*}}-\frac{Q}{Q^{*}}+\ln \left(\frac{Q}{Q^{*}}\right)-\ln \left(\frac{I}{I^{*}}\right)\right)=: a_{43} G_{43} \\
& D_{5}^{\prime}=\left(1-\frac{R^{*}}{R}\right)\left(\alpha_{r} I+\psi Q-(\omega+\mu) R\right) \\
& \leq \alpha_{r} I^{*}\left(\frac{I}{I^{*}}-\frac{R}{R^{*}}+\ln \left(\frac{R}{R^{*}}\right)-\ln \left(\frac{I}{I^{*}}\right)\right) \\
& +\psi Q^{*}\left(\frac{Q}{Q^{*}}-\frac{R}{R^{*}}+\ln \left(\frac{R}{R^{*}}\right)-\ln \left(\frac{Q}{Q^{*}}\right)\right)=: a_{53} G_{53}+a_{54} G_{54}
\end{aligned}
$$

where $a_{13}=a_{21}=\frac{\beta S^{*} I^{*}}{N}, a_{15}=\omega R^{*}, a_{32}=\gamma E^{*}, a_{34}=k Q^{*}, a_{43}=\alpha_{q} I^{*}, a_{53}=\alpha_{r} I^{*}$, $a_{54}=\psi Q^{*}$ and all other $a_{i j}=0$. The associated weighted digraph $(G, A)$ has five vertices and two cycles as shown in figure 2 Along each cycle, $G_{32}+G_{23}=0$ and $G_{34}+G_{43}=0$. By Proposition 1.3 of [12], there exists $c_{i}, 1 \leq i \leq 4$, such that $V=\sum_{i=1}^{5} c_{i} D_{i}$ is a Lyapunov function for equations (1)-(5). The relations between $c_{i}$ 's can be derived from Theorems 3.3 and 3.4 of [12] such that $d^{+}(1)=1$ implies $c_{1}\left(a_{15}+a_{13}\right)=c_{2} a_{21}, d^{+}(2)=1$ implies $c_{3} a_{32}=c_{2} a_{21}+c_{2} a_{23}, d^{-}(4)=1$ implies $\left.c_{4} a_{43}=c_{3} a_{34}+c_{5} a_{54}\right)$, and $d^{+}(5)=1$ implies $c_{5}\left(a_{54}+a_{53}\right)=c_{1} a_{15}$. Hence, $c_{2}=$ 
$\left(\frac{a_{15}+a_{13}}{a_{21}}\right) c_{1}, c_{3}=\left(\frac{\left(a_{15}+a_{13}\right)\left(a_{23}+a_{21}\right)}{a_{32}}\right) c_{1}, c_{4}=\left(\frac{\left(a_{15}+a_{13}\right)\left(a_{23}+a_{21}\right) a_{34}}{a_{32} a_{21}}+\frac{a_{15} a_{54}}{a_{54}+a_{53}}\right) c_{1}$ and $c_{5}=\left(\frac{a_{15}}{a_{54}+a_{53}}\right) c_{1}$. Therefore, $V=c_{1} D_{1}+c_{2} D_{2}+c_{3} D_{3}+c_{4} D_{4}+c_{5} D_{5}$ is a Lyapunov function for (1)- (5). Therefore, $E^{\star}$ is globally asymptotically stable in the interior of $\Omega$ when $\mathscr{R}_{0}>1$.

\section{Parameter values, elasticity indices and numerical simulations}

\subsection{Parameter values}

Table 1 shows parameter values used in this paper, as explained in Table 1 and sections 2.2 and 2.3 of [6] and Table 1 of [5]. Since the life expectancy of cattle is in average 5 years, the value of $\mu$ is taken to be $\frac{1}{5 \times 365}$. As indicated in [5,6], the incubation period, is between 4 and 8 weeks, we use the mean value of 6 weeks, $\gamma=\frac{1}{6 \times 7}$, the infection period is between 6 and 10 weeks, we used $\alpha_{r}=\frac{1}{8 \times 7}$ as mean value of 8 weeks. The persistently infected period is given in a range of $18-21$ weeks, with an average period of 19 weeks, 4 months $\times 2$ reactivations per month for 582 cases gives $k=0.0009$ and $\psi=0.0075$. And the rate of loss of natural immunity, $\omega=\frac{1}{3703}$. Maximum and minimum values are as in [5, 6].

\subsection{Elasticity indices}

We note from Theorems 3 and 4 that $\mathscr{R}_{0}$ plays an important role in determining the persistence of the disease in the cattle population under consideration. Thus, it is crucial to determine the sensitivity of $\mathscr{R}_{0}$ to each parameter. The elasticity of $\mathscr{R}_{0}$ relative to the parameter $p$ is given by the formula $\frac{p}{\mathscr{R}_{0}} \frac{\partial \mathscr{R}_{0}}{\partial p}$.

Table 2 shows the elasticity indices of $\mathscr{R}_{0}$ relative to those parameters in descending order. In a paper that developed a homogeneous, stochastic, compartmental model for CBPP transmission in pastoral herds of East Africa [6], a sensitivity analysis was completed using linear regression to estimate the impact of all input parameters on the total number of cases; the total number of cases was inversely related to the CBPP mortality rate, $\sigma(-0.755)$, and by positive relationship with the effective contact rate, $\beta(0.262)$, and the natural mortality rate, $\mu(0.157)$ and less sensitive to initial exposed, $E_{0}(-0.082)$, initial recovered, $\mathscr{R}_{0}(-0.071)$, the rate of sequestra formation, $\alpha_{q}(0.053)$, and the rate of recovery of persistently infected, $\psi(0.049)$. The regression model for total cases had an $r^{2}$ of 0.654 . The persistence of infection was also highly sensitive to $\sigma(-0.803)$ and moderately sensitive to $\alpha_{q}(0.234)$, 


\begin{tabular}{|c|c|c|c|}
\hline Parameter & Description & $\begin{array}{l}\text { Baseline } \\
\text { value }\end{array}$ & $\begin{array}{l}\text { Value range with time } \\
\text { unit }=1 \text { day } \\
\text { and references }\end{array}$ \\
\hline$\alpha_{q}$ & $\begin{array}{l}\text { Rate of sequestrum } \\
\text { formation }\end{array}$ & 0.013 & 0.011 to $0.018|6|$ \\
\hline$\alpha_{r}$ & Rate of recovery & $\frac{1}{8 \times 7}$ & $\frac{1}{10 \times 7}$ to $\frac{1}{6 \times 7}|6|$ \\
\hline$\beta$ & $\begin{array}{l}\text { Effective contact } \\
\text { rate }\end{array}$ & 0.126 & 0.07 to $0.13|6|$ \\
\hline$\gamma$ & $\begin{array}{l}\text { Transition rate } \\
\text { from exposed to } \\
\text { infectious } \\
\text { compartment }\end{array}$ & $\frac{1}{6 \times 7}$ & $\frac{1}{4 \times 7}$ to $\frac{1}{8 \times 7}|6|$ \\
\hline$k$ & $\begin{array}{l}\text { Rate of sequestrum } \\
\text { reactivation }\end{array}$ & 0.00009 & $|0.00007-0.00011| 6 \mid$ \\
\hline$\mu$ & Mortality rate & $\frac{1}{5 \times 365}$ & $\begin{array}{l}\frac{1}{6 \times 365} \text { to } \frac{1}{4.5 \times 365} \\
|6| \text { and estimated guest }\end{array}$ \\
\hline$\omega$ & $\begin{array}{l}\text { Rate of loss of } \\
\text { natural immunity }\end{array}$ & $\frac{1}{3703}$ & $|6|$ \\
\hline$\psi$ & $\begin{array}{l}\text { Rate of sequestrum } \\
\text { resolution }\end{array}$ & 0.0075 & 0.0068 to $0.0079|6|$ \\
\hline
\end{tabular}

Table 1 Description of model parameters and their values (in day $^{-1}$ ); indicating baselines, ranges and references.

$\beta(0.091)$ and $\gamma(0.072)$ with an $r^{2}$ of 0.704 . When the herd size was reduced to 500 head, the sensitivity profile changed; total cases was sensitive to $\gamma(-0.175)$, $\beta(0.149), \alpha_{r}(-0.145)$ and $\sigma(-0.129)$ but the $r^{2}$ of the regression model was only 0.100 . The persistence of infection was sensitive to $\gamma(-0.164)$ and $\psi(0.108)$ with an $r^{2}$ of only $0.040[6]$.

\begin{tabular}{|l|l|l|l|}
\hline Parameter $(p)$ & Formula: $\frac{p}{\mathscr{R}_{0}} \frac{\partial \mathscr{R}_{0}}{\partial p}$ & $\begin{array}{l}\text { Value of the } \\
\text { Parameter }\end{array}$ & $\begin{array}{l}\text { Elasticity } \\
\text { index }\end{array}$ \\
\hline$\beta$ & 1 & 0.126 & 1 \\
\hline$\alpha_{r}$ & 0.017857 & -0.57122 \\
\hline$\alpha_{q}$ & $\frac{-\alpha_{r} \bar{\mu}_{k \psi}}{\bar{\mu}_{k \psi} \bar{\mu}_{\alpha}-\alpha_{q} k}$ & 0.013000 & -0.41585 \\
\hline$\mu$ & $-\frac{\alpha_{q} \mu_{k \psi}}{\bar{\mu}_{k \psi}\left(\bar{\mu}_{\alpha}-\alpha_{q} k\right)}$ & 0.00054795 & 0.13001 \\
\hline$\gamma$ & $\frac{\mu}{\bar{\mu}_{k \psi}}-\frac{\mu}{(\gamma+\mu)}$ & 0.023810 & 0.022496 \\
\hline$k$ & $1-\frac{\gamma}{(\gamma+\mu)}$ & 0.0045481 \\
\hline$\psi$ & $\frac{k}{\bar{\mu}_{k \psi}} \frac{\alpha_{q}(\mu+\psi)}{\left(\bar{\mu}_{k \psi} \bar{\mu}_{\alpha}-\alpha_{q} k\right)}$ & 0.00009 & \\
\hline & $+\frac{\mu\left(\bar{\mu}_{k \psi}+\bar{\mu}_{\alpha}\right)}{\left(\bar{\mu}_{k \psi} \bar{\mu}_{\alpha}-\alpha_{q} k\right)}$ & & -0.0042385 \\
\hline & $\frac{\psi}{\bar{\mu}_{k \psi}}-\frac{\psi \bar{\mu}_{\alpha}}{\bar{\mu}_{k \psi} \bar{\mu}_{\alpha}-\alpha_{q} k}$ & 0.0075 & \\
\hline
\end{tabular}

Table 2 Elasticity indices of $\mathscr{R}_{0}$ relative to the chosen parameters. 


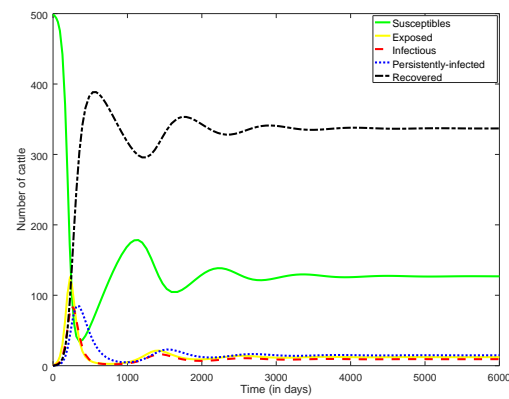

Fig. 3 The graph showing the number of cattle in each compartment using parameters in Table 1 with $I=1, N=500$, yielding an approximated equilibrium value $S^{*}=127, E^{*}=$ $12, I^{*}=9, Q^{*}=15, R^{*}=337$ and $\mathscr{R}_{0}=3.9399$.

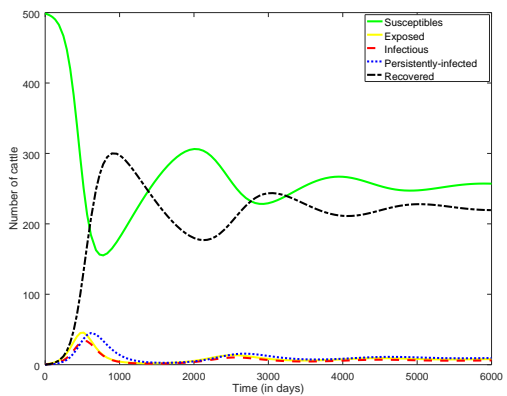

Fig. 4 The graph showing the number of cattle in each compartment when $\beta=0.07$ and the other parameters are as in Table 1 with $I=$ $1, N=500$, yielding the approximated equilibrium of $S^{*}=257, E^{*}=8, I^{*}=6, Q^{*}=9, R^{*}=$ 220 and $\mathscr{R}_{0}=1.9699$.

From Table 2 , effective contact rate $(\beta)$ has the largest elasticity index followed by and the rate of recovery of infectious cattle $\left(\alpha_{r}\right)$. the rate of sequestrum formation $\left(\alpha_{q}\right)$ takes the third, the mortality rate the fourth position. Followed by the incubation rate $\gamma$, the rate of sequestrum reactivation $(k)$ and the rate of recovery of persistently infected cattle $(\psi)$, respectively. Which implies that $\beta$ and $\alpha_{q}$ affects the basic reproduction number more followed by $\psi$ and $\alpha_{r}$, respectively.

\subsection{Numerical simulations}

We consider a constant herd size of 500 cattle with individual animals as the epidemiological units of interest. Assuming the presence of an infectious cattle and 499 susceptible cattle initially in the cattle population, the model equations (1) - (5) are numerically solved using the parameter values given in Table 1 With these values, $\mathscr{R}_{0}=3.9399$. The number of cattle in each compartment at time $t$ are plotted in Figure 3 in which the system approaches an endemic equilibrium as $\mathscr{R}_{0}>1$. These values are in agreement with results published in [5,6]. Since the effective contact rate $\beta$ has the largest elasticity index, we decrease the value of $\beta$ by $50 \%$, assuming half of the transmissions, the results suggest that double of the cattle remain susceptible and the basic reproduction number is reduced by half, this is shown in Figure 4 This suggests that quarantine should be the primary measure to try to control the disease. However, as proposed in [5, 6, 10], this will not be sufficient to eradicate the disease. 


\section{Conclusion and Remarks}

In this paper we derived a formula for the basic reproduction number $\mathscr{R}_{0}$ for our model of CBPP transmission. By using a matrix theoretic method and a graph theoretic method respectively, we prove that for $\mathscr{R}_{0} \leq 1$, the DFE is globally asymptotically stable, thus CBPP dies out; whereas for $\mathscr{R}_{0}>1$, the EE is globally asymptotically stable and hence the disease persists in all the populations.

Using parameter values we calculate elasticity indices for $\mathscr{R}_{0}$ with respect to different model parameters. Based on our numerical elasticity indices, $\mathscr{R}_{0}$ is very sensitive to the effective contact rate $\beta$; which has also large regression coefficient in [18], the rate of recovery of persistently infected cattle $\psi$; in contrary, it has less regression coefficient in [6] and the rate of recovery of infectious cattle $\alpha_{r}$; but less sensitive to the death/birth rate $\mu$; which has large regression coefficient in [18], the rate of infection reactivation of persistently infected cattle $k$, and the rate of transition from exposed to infectious class $\gamma$. Which implies that for some value of these parameters, $\mathscr{R}_{0}$ becomes less than 1 ; thus the disease can be controlled. In addition, if we apply vaccination and/or restriction of movement along with treatment using antibiotics to the extent that reduce the value of $\beta$, and increase the values of $\alpha_{r}$ and $\alpha_{q}$ so that $\mathscr{R}_{0}$ becomes less than 1 . However, there seems to be no way to manage $\psi$.

From our simulations for the given parameter values, the number of infectious cattle is about $2 \%$ of the population at equilibrium. Our model ignores death due to CBPP. However, our elasticity indices give an indication that CBPP can be controlled by reducing the value of $\beta$; which refers to the use of vaccination and/or applying restriction of movement of cattle; however, it is well known that the vaccine provides only short-term immunity, can cause severe adverse reactions and is rarely effective in the face of a severe outbreak, or/and by increasing the values of $\alpha_{r}$ and $\alpha_{q}$; which is achieved by treating cattle using antibiotics; though treatment of infected cattle with antibiotics can result in healthy looking animals that are still infected and able to spread the disease, so it is not recommended. However, the vaccination of healthy animals combined with treatment of clinical cases is proposed to be the most promising intervention scenario [5].

\section{References}

1. N. Abdela and N. Yune. Seroprevalence and Distribution of Contagious Bovine Pleuropneumonia in Ethiopia: Update and Critical Analysis of 20 Years (1996-2016) Reports. Frontiers in veterinary science, 4:100, 2017.

2. V. Dupuy, L. Manso-Silván, V. Barbe, P. Thebault, E. Dordet-Frisoni, C. Citti, F. Poumarat, A. Blanchard, M. Breton, P. Sirand-Pugnet, and F. Thiaucourt. Evolutionary History of Contagious Bovine Pleuropneumonia Using Next Generation Sequencing of Mycoplasma mycoides Subsp. mycoides Small Colony. PLoS ONE, 7(10):e46821, oct 2012.

3. J. Jores, J. C. Mariner, and J. Naessens. Development of an improved vaccine for contagious bovine pleuropneumonia: An African perspective on challenges and proposed actions. Veteri- 
nary Research, 44(1-5):122, 2013.

4. M. Lesnoff, G. Laval, P. Bonnet, and A. Workalemahu. A mathematical model of contagious bovine pleuropneumonia ( CBPP ) within-herd outbreaks for economic evaluation of local control strategies : an illustration from a mixed crop-livestock system in Ethiopian highlands. Animall Research, EDP Sciences, 53:429-438, 2004.

5. J. C. Mariner, J. McDermott, J. A. Heesterbeek, G. Thomson, P. L. Roeder, and S. W. Martin. A heterogeneous population model for contagious bovine pleuropneumonia transmission and control in pastoral communities of East Africa. Preventive Veterinary Medicine, 73(1):75-91, 2006.

6. J. C. Mariner, J. McDermott, J. A. P. Heesterbeek, G. Thomson, and S. W. Martin. A model of contagious bovine pleuropneumonia transmission dynamics in East Africa. Preventive veterinary medicine, 73(1):55-74, 2006.

7. G. Muuka, N. Songolo, S. Kabilika, B. M. Hang'ombe, K. S. Nalubamba, and J. B. Muma. Challenges of controlling contagious bovine pleuropneumonia in sub-Saharan Africa. Tropical Animal Health and Production, 45(1):9-15, dec 2012.

8. R. Nicholas, R. Ayling, and L. Mcauliffe. Mycoplasma diseases of ruminants. CABI, 1-239. 10.1079/9780851990125.0000, 2008.

9. J. O. Onono, B. Wieland, and J. Rushton. Estimation of impact of contagious bovine pleuropneumonia on pastoralists in Kenya. Preventive Veterinary Medicine, 115(3-4):122-129, aug 2014.

10. M. J. Otte, R. Nugent, and A. Mcleod. Transboundary Animal Diseases: Assessment of socioeconomic impacts and institutional responses Food and Agriculture Organization. Technical report, Transboundary animal diseases: Assessment of socio-economic impacts and institutional responses, FAO, Rome, 2004.

11. F. Sacchini, M. Luciani, R. Salini, M. Scacchia, A. Pini, R. Lelli, J. Naessens, J. Poole, and J. Jores. Plasma levels of TNF- $\backslash$ alpha, IFN- $\backslash$ gamma, IL-4 and IL-10 during a course of experimental contagious bovine pleuropneumonia. BMC Veterinary Research, 8(1):44, apr 2012.

12. Z. Shuai and P. van den Driessche. Global stability of infectious disease models using Lyapunov functions. SIAM Journal on Applied Mathematics, 73(4):1513-1532, 2013.

13. H. L. Smith and P. Waltman. The Theory of the Chemostat: Dynamics of Microbial Competition. Cambridge University Press, Cambridge, 1995.

14. A. Ssematimba, J. Jores, and J. C. Mariner. Mathematical Modelling of the Transmission Dynamics of Contagious Bovine Pleuropneumonia Reveals Minimal Target Profiles for Improved Vaccines and Diagnostic Assays. PLOS ONE, 10:e0116730, feb 2015.

15. N. E. Tambi, W. O. Maina, and C. Ndi. An estimation of the economic impact of contagious bovine pleuropneumonia in Africa. Revue scientifique et technique (International Office of Epizootics), 25(3):999-1011, dec 2006.

16. P. van den Driessche and J. Watmough. Reproduction numbers and sub-threshold endemic equilibria for compartmental models of disease transmission. Mathematical biosciences, 180:29-48, 2002.

17. E. M. Vilei and J. Frey. Detection of Mycoplasma mycoides subsp. mycoides SC in bronchoalveolar lavage fluids of cows based on a TaqMan real-time PCR discriminating wild type strains from an $\mathrm{lppQ}^{\wedge}\{-\}$ mutant vaccine strain used for DIVA-strategies. Journal of microbiological methods, 81(3):211-218, jun 2010.

18. R. S. Windsor. The Eradication of Contagious Bovine Pleuropneumonia from South Western Africa: A Plan for Action. Annals of the New York Academy of Sciences, 916(1):326-332, jan 2000 . 